\title{
The touchscreen cognitive testing method for rodents: How to get the best out of your rat
}

\author{
Timothy J. Bussey, ${ }^{1,2,4}$ Tina L. Padain, ${ }^{1,3}$ Elizabeth A. Skillings, ${ }^{1,3}$ Boyer D. Winters, ${ }^{1}$ \\ A. Jennifer Morton, ${ }^{3}$ and Lisa M. Saksida ${ }^{1,2}$ \\ ${ }^{1}$ Department of Experimental Psychology, University of Cambridge, Cambridge CB2 3EB, United Kingdom; ${ }^{2}$ The MRC and
Wellcome Trust Behavioural and Clinical Neuroscience Institute, University of Cambridge, Cambridge CB2 3EB, United Kingdom;
${ }^{3}$ Department of Pharmacology, University of Cambridge, Cambridge CB2 1PD, United Kingdom
}

The touchscreen testing method for rodents is a computer-automated behavioral testing method that allows computer graphic stimuli to be presented to rodents and the rodents to respond to the computer screen via a nose-poke directly to the stimulus. The advantages of this method are numerous; however, a systematic study of the parameters that affect learning has not yet been conducted. We therefore sought to optimize stimuli and task parameters in this method. We found that when parameters were optimized, Lister Hooded rats could learn rapidly using this method, solving a discrimination of two-dimensional stimuli to a level of $80 \%$ within five to six sessions lasting $\sim 30$ min each. In a final experiment we tested both male and female rats of the albino Sprague-Dawley strain, which are often assumed to have visual abilities far too poor to be useful for studies of visual cognition. The performance of female Sprague-Dawley rats was indistinguishable from that of their male counterparts. Furthermore, performance of male Sprague-Dawley rats was indistinguishable from that of their Lister Hooded counterparts. Finally, Experiment 5 examined the ability of Lister Hooded rats to learn a discrimination between photographic stimuli. Under conditions in which parameters were optimized, rats were remarkably adept at this discrimination. Taken together, these experiments served to optimize the touchscreen method and have demonstrated its usefulness as a high-throughput method for the cognitive testing of rodents.

It is now well understood that the use of animal models is an essential component in our quest to understand the brain mechanisms of cognition. In addition, such models are necessary for researchers to be able to test potential therapeutic agents in a direct and expedient manner that is not possible in human subjects. Moreover, we are experiencing a revolution in the sophistication of transgenic and knock-out animal models that creates opportunities for cognitive testing and therapeutic screening that were until recently, unthinkable (Kobayashi and Chen 2005).

To achieve successful translation of findings from animal models to humans, the cognitive tasks we use with our rodents should resemble as closely as possible those used with human subjects. Increasingly, human subjects are tested using computerautomated methods. A particularly powerful approach is the "touchscreen" testing method, in which subjects respond directly to stimuli on a computer screen (e.g., Robbins et al. 1994). The advantages of this method are numerous and include facilitated performance due to contiguity between stimuli and responses and the ability to present a battery of different cognitive tests in which parameters such as stimuli, responses, and feedback are consistent across tasks and conditions.

To create a cognitive testing method for rodents that matched, as far as possible, the touchscreen testing method for humans, Bussey et al. (1994, 1997a,b) (see Fig. 1A) developed the touchscreen testing method for rats, a method which has more recently been adapted for mice (Izquierdo et al. 1993; Bussey et al. 2001b; Brigman et al. 2005; Morton et al. 2006). This computer-automated behavioral testing method allows computer graphic stimuli to be presented to a rodent and the rodent to respond to the computer screen via a nose-poke directly to the stimulus. The nose-poke response is detected by an infrared

\footnotetext{
${ }^{4}$ Corresponding author.
}

E-mail tjb1000@cam.ac.uk; fax 44-(0)-1223-333564.

Article is online at http://www.learnmem.org/cgi/doi/10.1101/lm.987808. touchscreen assembly surrounding the screen (see Fig. 1). In addition to the advantages of facilitated translation between human and animal tests, the task offers many other advantages, including low motoric demand, ease of administration, and accuracy of task parameters. The method has been used effectively in a number of behavioral, lesion, and pharmacological studies (e.g., Izquierdo et al. 1993; Sahgal and Steckler 1994; Steckler and Sahgal 1995; Markham et al. 1996; Bussey et al. 1997a,b, 1998, 2001a; Parkinson et al. 1999, 2002; Chudasama et al. 2001; Chudasama and Robbins 2003; Cook et al. 2004; Dalley et al. 2005; Janisewicz and Baxter 2003; Brigman et al. 2005; Minini and Jeffery 2006; Morton et al. 2006).

Despite the effectiveness of this method as it is presently used, a systematic study of the parameters that affect learning has not yet been conducted. Such optimization would pay high dividends, as one advantage of such an automated method is that it can provide a relatively high-throughput method of cognitive testing, and therefore the more rapid the learning the better. In this study, therefore, we examined a number of stimulus and other task parameters with a view to optimizing the procedure. In Experiment 1, we assessed a number of stimuli for "biases," that is, the preference of rats for one stimulus over another with which it is paired. Such spontaneous biases create problems of interpretation and add variability to the data, and therefore should be minimized. Having identified stimulus pairs with low biases, in Experiment 2 we used several of these pairs to examine the effects of stimulus dimensions on the rate of learning, by comparing the learning rates of rats discriminating pairs of stimuli of different sizes. In Experiment 3, we turned from stimulus parameters to other task parameters, namely the length of the inter-trial interval (the period between trials), the number of trials per daily session (small versus large), and the requirement for the rat to perform a trial initiation response, as opposed to trials initiating automatically. Whereas these experiments were carried 
A

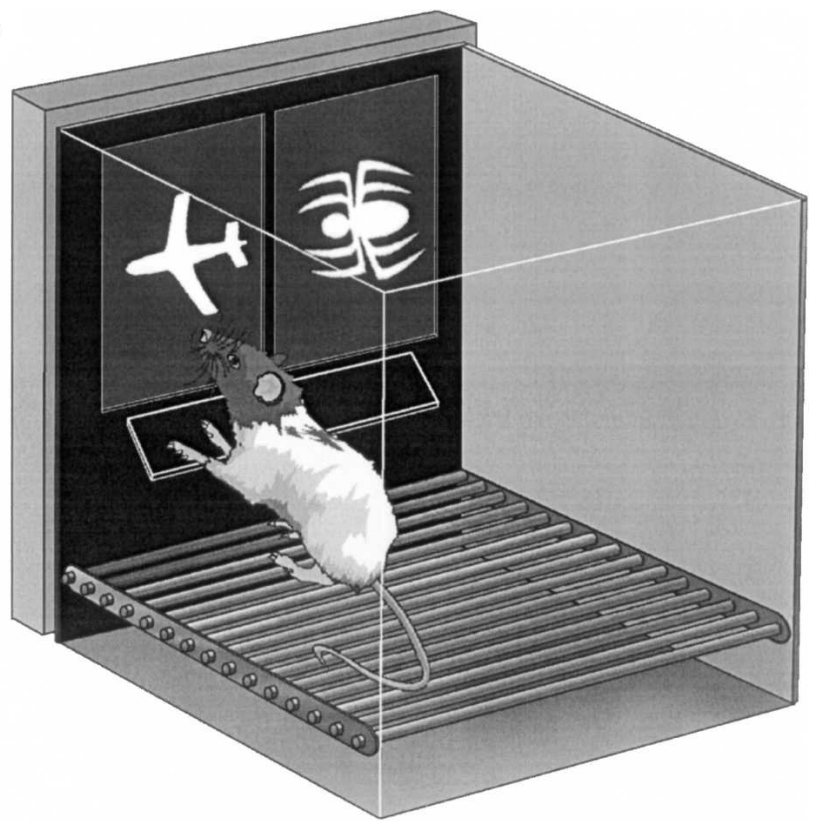

B

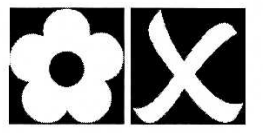

Stimulus Pair 1

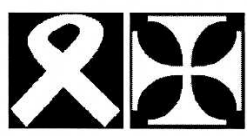

Stimulus Pair 4

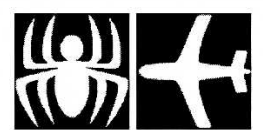

Stimulus Pair 2

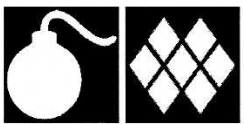

Stimulus Pair 5

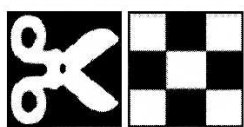

Stimulus Pair 3

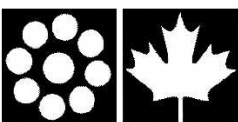

Stimulus Pair 6

Figure 1. (A) The rat touchscreen apparatus. One wall of a standard operant chamber was removed and replaced with a flat-screen monitor and touchscreen assembly. A Perspex "mask" was placed over the screen; this was a sheet of Perspex that covered the screen with two response windows through which the rat could make a nose-poke toward the screen. Attached to the mask was a "shelf," the effect of which was to force the rat to stop, rear up, and stretch toward the stimuli with a head-on approach, thus facilitating the rat's attention to the stimuli. The magazine containing built-in photocells was located on the rear wall (not shown). (B) Pairs of stimuli used in Experiment 1. Pairs 1, 2, 3, and 6 were used in Experiment 2. Pair 2 was used for Experiments 3 and 4.

out using male rats of the Lister Hooded strain, in a subsequent experiment we tested both male and female rats of the albino Sprague-Dawley strain, which are often assumed to have visual abilities far too poor to be useful for studies of visual cognition (albino rats' acuities are about half that of pigmented strains; Prusky et al. 2002). Female rats were included because many researchers have reason to focus their studies on female rats. The inclusion of Sprague-Dawley and female rats provided a particularly stringent test of the utility of the optimized method. Finally, because Experiments 1-4 were carried out using the same type of visual stimuli, namely white shapes on a black background, Experiment 5 examined the ability of Lister Hooded rats to learn a discrimination between complex photographic stimuli.

\section{Results}

Experiment 1: Identifying and minimizing spontaneous stimulus bias

It is well known that rodents exhibit preferences for some stimuli over others. This characteristic of rodent behavior can be prob-

lematic for cognitive testing, particularly in forced-choice discrimination tasks, in which the rodent may spontaneously and repeatedly select one stimulus over another from the outset of behavioral testing. It then becomes difficult to determine whether the effects of a manipulation such as a drug or a lesion are due to the manipulation affecting the task per se or to the expression of a stimulus bias. In Experiment 1, therefore, we first identified several pairs of stimuli (Fig. 1B) and tested for spontaneous biases by presenting them to rats in a single pairwise discrimination session. The results of bias testing are shown in Figure 2. Dotted lines indicate significant deviation from chance (50\%) according to the binomial distribution. As can be seen from the figure, only one of the six stimulus pairs tested yielded a significant bias (pair 4), rats showing a spontaneous preference for one of the two stimuli (the "ribbon," the left stimulus in pair 4 in Fig. 1B).

\section{Experiment 2: Optimizing stimulus size}

In this experiment, we sought to determine the optimal size of visual stimuli for rats in the touchscreen method. In previous touchscreen studies, the size of the visual stimuli we used was determined more or less arbitrarily. Thus, we systematically analyzed the performance of rats tested with different sizes of stimuli to determine what stimulus size is closest to optimal. In Experiment 1 , we identified five pairs of stimuli that yielded acceptable bias scores. Four of these stimulus pairs (pairs 1,2, 3, and 6) were chosen arbitrarily and used in Experiment 2 for size optimization. Results of size testing are shown in Figure 3, which indicates that size 4 (the largest size) yielded the most rapid acquisition and the highest performance levels. ANOVA revealed a significant main effect of size $\left(F_{(3,80)}=5.18, P<0.005\right)$. Tukey post-hoc analysis revealed that this effect was due to the rats in size groups 1 and 3 performing at significantly lower levels than those in size group 4 . No significant difference in performance was found between size groups 4 and 2, but this can probably be attributed to the similar rates of learning in these groups over the first four days of the discrimination, after which performance diverged. There was no significant size $\times$ stimulus interaction $(F<1)$ nor was there an effect of stimulus pair $\left(F_{(3,28)}=1.00, P=0.41\right.$.)

\section{Experiment 3: Optimizing inter-trial interval, trials per} session, and trial initiation requirements

Behavioral experiments have shown that learning proceeds more rapidly when inter-trial intervals (ITIs) are long than when they are short (e.g., Barnet et al. 1995). Therefore, in Experiment 3, we compared the rate of learning with two different ITIs, $5 \mathrm{sec}$ (the ITI used in most previous touchscreen experiments) and a longer $20 \mathrm{sec}$. It was predicted that the introduction of a longer ITI might lead to an improved learning rate. We also varied the

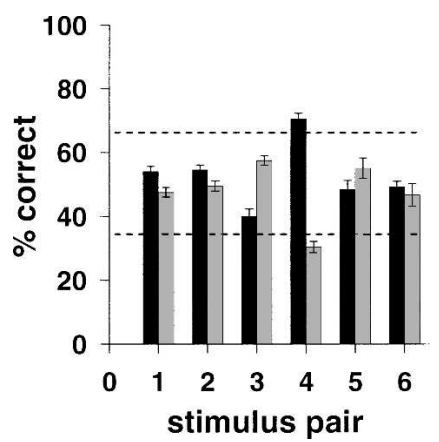

Figure 2. Results of bias tests (Experiment 1) for the six stimulus pairs. Rats showed a significant bias to respond to one of the two stimuli in pair 4 only. 


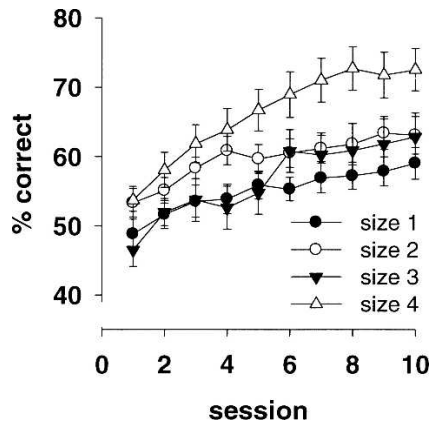

Figure 3. Acquisition curves of rats performing visual discriminations with different stimulus sizes (Experiment 2). Rats acquired the discrimination most rapidly when the largest stimuli were used.

number of trials per session. Although it seems obvious that more trials should lead to more learning, it may be that later trials in a session lead to "diminishing returns" and that the advantages of large numbers of trials per session might be outweighed by the increase in the time it takes to complete a session. In addition, if it did transpire that longer ITIs were desirable, then in future studies it may be worth reducing the number of trials, if possible, to increase the ITI while keeping the session length optimized. (Previous studies have generally used up to 100 trials with an ITI of $5 \mathrm{sec}$ for rats [Bussey et al. 1994, 1997b] or 20-30 trials with ITIs of 5 sec or shorter for the mouse [Bussey et al. 2001b; Morton et al. 2006].) Finally, the touchscreen method as previously used includes a requirement for rodents to initiate a trial-that is, to bring up the choice stimuli following the ITI-by returning to the rear of the testing chamber either to stand on a pressuresensitive platform (Bussey et al. 1994), to make a nose-poke to the magazine (this study), or to press a lever (Bussey et al. 2001b). The rationale for this requirement is that it brings the animal to the back of the testing chamber, thus minimizing accidental stimulus choices, and allows a good view of the stimulus display, thereby maximizing stimulus sampling time. It is not known, however, if the requirement to initiate really has any effect on the rate of learning. This is an important consideration, because if the trial initiation requirement has little effect on performance, there is no reason to devote time to pretraining the trial initiation response. In this experiment, we tested rats in two conditions, one in which they were required to initiate trials via a nose-poke to the magazine and one in which they were not. These three factors-ITI, trials per session, and trial initiation requirement-were examined in a fully counterbalanced within-subjects design. The results are shown in Figure 4. A nested, three-way ANOVA revealed a main effect of session $\left(F_{(20,1040)}=27.24, P<0.001\right)$. In addition, the groups trained with an initiation requirement performed significantly higher than the group with no initiation requirement (Fig. 4B) $\left(F_{(1,52)}=11.30, P<0.001\right)$, the groups with sessions consisting of 60 trials performed significantly higher than those with 20 trials (Fig. 4 C) $\left(F_{(1,52)}=5.66, P<0.05\right)$, and the groups trained with a long ITI showed a significantly higher performance than those trained with a short ITI (Fig. $4 \mathrm{D})\left(F_{(1,52)}=5.26, P<0.05\right)$. Thus, longer ITIs, more trials, and a requirement to initiate trials all lead to improved learning.

\section{Experiment 4: Sex and strain comparisons- The performance of male and female albino} Sprague-Dawley rats on discrimination and reversal A further question in which we were interested is the general applicability of the rat touchscreen method. Experiments 1-3 used male rats of the Lister Hooded strain. In this experiment, we sought to compare, on both acquisition of the discrimination and reversal (when the $S+$ becomes the $S$ - and vice versa), the performance of male and female rats. Testing whether female rats perform well in this apparatus is important, as many researchers have reason to use female rats. We also compared the performance of Lister Hooded rats with those of the Sprague-Dawley strain. These rats are albino and have much lower visual acuity $(\sim 0.5$ cycles per degree) than non-albino strains (Prusky et al. 2002). Many researchers have reason to use this strain, but it is widely assumed that their relatively poor acuity renders them unsuitable for testing visual cognition. The results of sex and strain comparisons are shown in Figure 5. For the sex comparison (Fig. 5A,B), animals were tested to a predetermined criterion; therefore, learning curves were constructed using only the sessions for which all rats' data could be included, and learning rates were compared by analyzing the number of sessions to criterion or each rat. For the strain comparison (Fig. 5C), rats were tested for a set number of days, and so both the learning curves and sessions to criterion were analyzed. A $t$-test carried out to analyze sessions to criterion scores comparing Sprague-Dawley males and females (Fig. 5A, right) revealed no significant difference between the two groups $\left(t_{(14)}=1.11, P=0.28\right.$, two-tailed). Similarly, the same test carried out to analyze sessions to criterion at the reversal stage (Fig. 5B, right) revealed no significant difference $\left(t_{(14)}=0.10, P=0.92\right.$, two tailed). These findings indicate that female rats are able to perform as well on these touchscreen tasks as their male counterparts. The data from male Sprague-Dawley rats was then compared with those obtained from male Lister Hooded rats in Experiment 3 (Fig. 5C, left). There was a main effect of session $\left(F_{(8,112)}=49.9, P<0.0001\right)$ but no session $\times$ group interaction $\left(F_{(8,112)}=1.34, P=0.23\right)$ and no main effect of group $\left(F_{(1,14)}=0.64, P=0.44\right)$. A $t$-test carried out to analyze sessions to criterion (Fig. $5 \mathrm{C}$, right) revealed no significant difference between the two groups $\left(t_{(14)}=1.75, P=0.10\right.$,

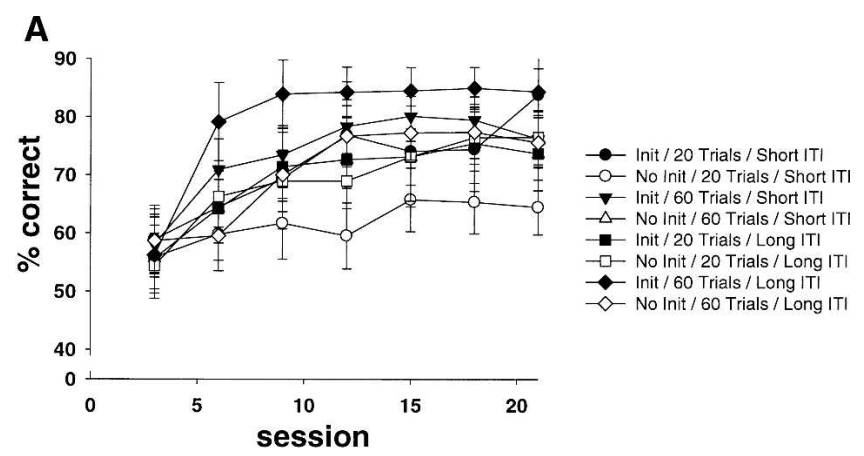

B

C

D

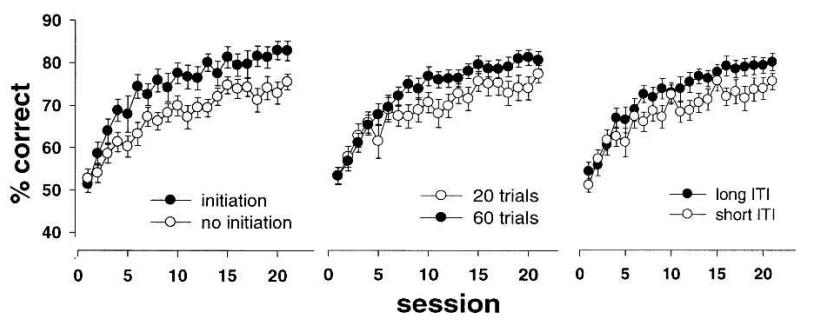

Figure 4. (A) Acquisition curves of rats performing visual discriminations with different combinations of task parameters. Data are plotted as blocks of three sessions. Rats were tested with all combinations of initiation or no initiation, 20 trials or 60 trials, and short or long ITI. A requirement for initiation $(B)$, a larger number of trials $(C)$, and a long ITI $(D)$ all significantly facilitated acquisition. 
A

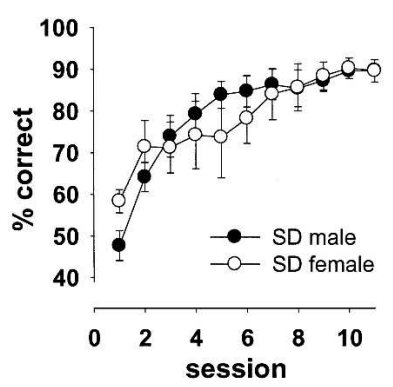

B

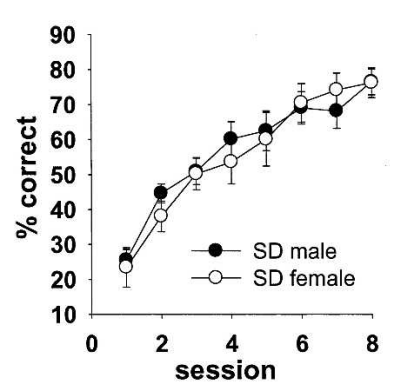

C

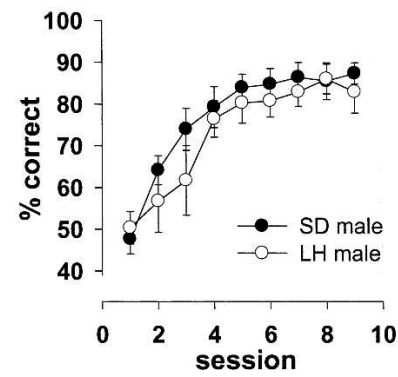

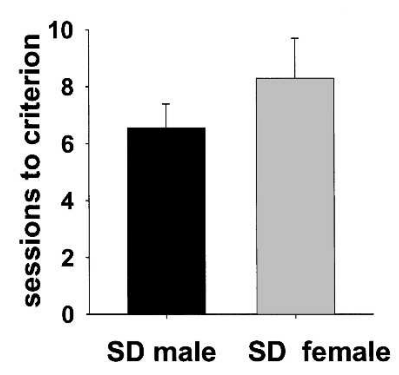
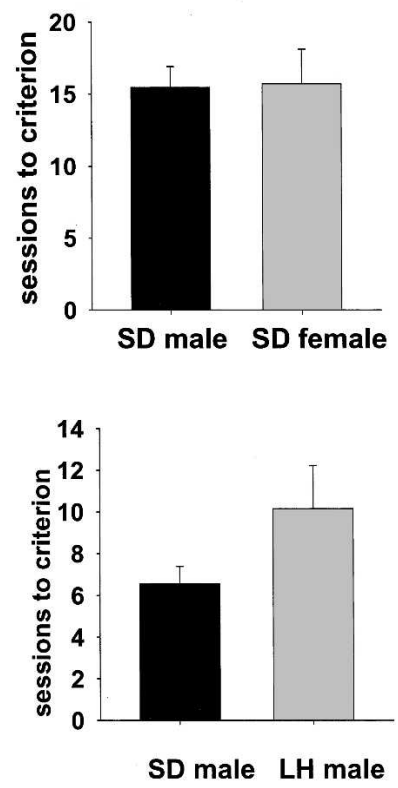

Figure 5. Learning curves and sessions to criterion for Sprague-Dawley male rats compared with those for Sprague-Dawley female rats (acquisition, $A$; reversal, $B$ ), and for Sprague-Dawley (SD) male rats compared with Lister Hooded (LH) male rats (C). All groups of rats acquired the task equally rapidly.

two-tailed), indicating that Sprague-Dawley rats, despite their lower visual acuity, are able to perform as well as their Lister Hooded counterparts.

\section{Experiment 5: Discrimination of photographic stimuli}

Experiments 1-4 were all carried out using the same type of visual stimuli, namely white shapes on a black background. Indeed, in all of our past experiments using both rats and mice, this type of stimulus was used. This raises the question of whether rodents are capable of discriminating other types of stimulus material in the touchscreen apparatus. Of particular interest is whether rodents can discriminate photographic stimuli, which are often used when testing human and non-human primates. The ability of rodents to discriminate such stimuli would thus provide even greater opportunity for comparison between species. Moreover, photographic stimuli are readily available in large numbers and can be manipulated in a number of different ways, for example using "morphing" technology to manipulate systematically the perceptual similarity of stimuli (Bussey et al. 2003). It might, however, be expected that rats, because of their poor visual acuity, would have considerable difficulty with such discriminations (Prusky et al. 2002). Furthermore, Minini and Jeffery (2006) have

suggested that rats solve discriminations presented on a touchscreen by using brightness differences in the lower areas of the screen that occur when elements of a shape stimulus protrude into these areas. If rats must solve discriminations in this manner, then two photographic stimuli of the same shape as each other should be quite difficult for rats to discriminate. In Experiment 5, therefore, we examined the ability of Lister Hooded rats to learn a discrimination between photographic stimuli. It was found that the rats performed remarkably well, attaining levels of above $80 \%$ correct within 14 sessions of 60 trials each (see Fig. $6 \mathrm{~A})$. ANOVA revealed a main effect of session $\left(F_{(15,210)}=37.5\right.$, $P<0.0001)$.

\section{Discussion}

The aim of this study was to optimize stimuli and task parameters in the touchscreen testing method for rodents. It was found that when parameters are optimized, rats can learn rapidly using this method, solving a discrimination of two-dimensional visual stimuli to a level of $80 \%$ within several sessions lasting about a half hour each. This rate of learning is as good as or better than that obtained using more labor-intensive "hand-testing" methods.

In Experiment 1, we evaluated the stimulus biases (spontaneous preferences) elicited by six pairs of shape stimuli. Biases were evaluated by giving one session of a rewarded discrimination in which one of the stimuli was designated the S+ (rewarded) and the other the $S$ - (unrewarded). As previous results indicate that little learning occurs within the first session of a discrimination using this method, spontaneous stimulus biases could be evaluated by recording the rats' scores during this session and evaluating the difference between these scores and a chance level score of $50 \%$ correct. Only one of the six stimulus pairs tested (pair 4) yielded a bias that was significantly different from chance. The finding that five of the six pairs tested yielded acceptable bias scores indicates that when these stimuli are used in this apparatus, problems with stimulus bias are minimal.

In Experiment 2, we used stimulus pairs 1, 2, 3, and 6 to evaluate whether the size of stimuli had an effect on the rate of learning, and if so, to determine the optimal stimulus size. Four
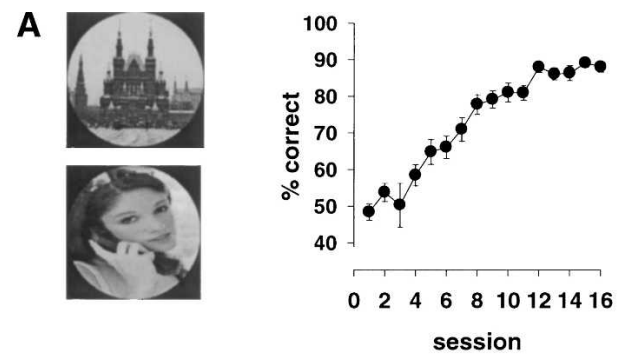

B

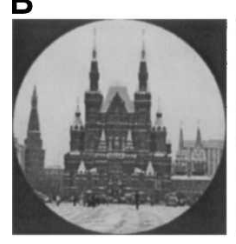

Original

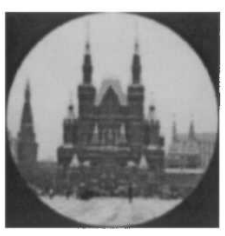

$2 \mathrm{~cm}$ distance

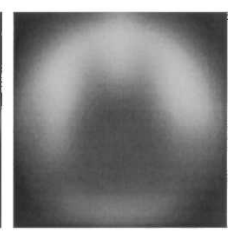

$40 \mathrm{~cm}$ distance
Figure 6. (A) Acquisition curve of rats performing a visual discrimination using photographic stimuli. Rats attained levels above $80 \%$ correct within 14 sessions of 60 trials each. (B) Photographic stimuli filtered to provide an approximation of how the stimuli might appear to rodents with a visual acuity of 1.0 cycle/degree, when viewing the stimuli from different distances. Based on the analysis by Prusky et al. (2002). 
sizes were evaluated, and it was found that the largest size stimulus-roughly $5.5 \mathrm{~cm}^{2}$-yielded the most rapid learning. As the stimulus size used in our previous studies (e.g., Bussey et al. 1994, 1997 b) was $\sim 2-3 \mathrm{~cm}^{2}$, corresponding roughly to our stimulus size 1 or 2 , this finding suggests that the learning rates published in these studies may have underestimated the visual learning abilities of the rat.

In Experiment 3, three factors-ITI, trials per session, and trial initiation requirement-were examined in a fully counterbalanced within-subjects design. It was found that the requirement for initiation, a greater number of trials, and long ITIs all led to significantly more rapid learning (Fig. 4B,C,D, respectively). Indeed, Figure 4A shows that numerically, the group of rats in which all three of these factors were combined acquired the discrimination at the fastest rate of the eight groups tested. It stands to reason that still longer ITIs and greater numbers of trials per session than those tested here might increase the learning rate even further. These parameters must be balanced, however, with the amount of time the rat remains in the testing chamber during a session. With parameters set at 60 trials and 20-sec ITI, a session lasts about $30 \mathrm{~min}$. This seems a reasonable session duration for both rat (in the apparatus as currently configured there is no source of water) and experimenter, but different researchers may have different priorities or requirements and may wish to adjust these parameters to suit their needs.

In Experiment 4, we tested rats of the Sprague-Dawley strain, which are often assumed to have visual abilities far too poor to be useful for studies of visual cognition (albino rats have an acuity of roughly 0.5 cycle per degree, whereas the value for pigmented rats is roughly twice that; see Prusky et al. 2002). We found that in the touchscreen apparatus, rats of this species performed well, indeed as well as did rats of the Lister Hooded strain. Finally, as many researchers have reason to focus their studies on female rats, we were interested in whether female rats could perform well using the touchscreen method. We tested this in a stringent manner, by comparing the learning rates of female albino Sprague-Dawley rats with their male counterparts. Female Sprague-Dawley rats' performances were found to be indistinguishable from those of the Sprague-Dawley males, which, as discussed above, learned as rapidly as did the Lister Hooded males. Although Lister Hooded females were not tested, the combined finding that female Sprague-Dawley rats' performances were indistinguishable from those of male Sprague-Dawley rats and that the male Sprague-Dawley rats' performances were indistinguishable from those of Lister Hooded males leads us to conclude that female rats of most strains could probably perform well using the touchscreen method.

Experiments 1-4 were carried out using the same type of visual stimuli, namely white shapes on a black background. In Experiment 5, we examined the ability of Lister Hooded rats to learn a discrimination between photographic stimuli. It was found that the rats performed remarkably well, attaining levels of $80 \%$ correct within 14 sessions of 60 trials each. As this type of stimulus material is often used when testing human and nonhuman primates, the finding that rats can discriminate such stimuli provides further opportunity for comparison between species. Moreover, photographic stimuli have several advantages over other types of stimuli, as they are readily available in large numbers and can be manipulated in a number of different ways, for example, using "morphing" technology to manipulate systematically the perceptual similarity of stimuli (Bussey et al. 2003).

The visual abilities of rats-and especially albino rats- have been much maligned; therefore, it may come as a surprise that rats can demonstrate such good performance on purely visual discriminations of two-dimensional stimuli, including photo- graphic stimuli, in this apparatus. One reason for this good performance may be the proximity of the stimulus to the rat's response, as it is known that increasing the spatial contiguity between stimulus and response facilitates learning (Stollnitz 1965). Consistent with this idea, Cook et al. (2004) found that rats learned a visual discrimination almost twice as rapidly when required to nose-poke the stimuli on a touchscreen as when responding to a lever situated beneath the stimuli. These authors suggest that the differential rates of learning are likely due to differences in the spatial contiguity of stimuli and responses, perhaps because attending to the location of the response draws resources away from the task of stimulus sampling. Similarly, Markham et al. (1996) found that rats learned in the touchscreen much more rapidly when reward delivery occurred at the rear of the testing chamber, away from the rat, as opposed to reward being delivered just underneath the stimuli. This result may seem surprising, since rats learned more rapidly when the spatial contiguity of stimulus and reward was poorest, but again, the poor performance in the condition under which reward was delivered near the stimulus may be due to rats attending to the site of reward delivery, rather than the stimulus, when it is making its nose-poke response. Indeed, in our initial pilot studies we, too, delivered food pellets into a cup just below the stimulus (T.J. Bussey, L.M. Saksida, and D.M. Wilkie, unpubl.) and observed that on choice rats did appear to orient toward the food cup rather than the stimuli, and therefore subsequent configurations of the apparatus delivered food reward to the rear of the chamber (Bussey et al. 1994).

Another factor that may facilitate learning in the touchscreen is that rats appear to make their choice of response when situated very close to the stimuli. Indeed, we have observed that well-trained rats approach the screen, rear up resting their paws on the shelf, and then make side-to-side head movements as they apparently scan both stimuli repeatedly before making a response. The visual acuity of rats is only about 1 cycle/degree (Prusky et al. 2002) and so viewing distance may make a significant difference in rats' abilities to discriminate stimuli. Indeed, Prusky et al. (2002) have provided an analysis in which photographic stimuli were filtered to provide an approximation to how the photographs might appear to different strains of rodents with different visual acuities. If we assume our rats to have a visual acuity of 1.0 cycle/degree, we can use the same manipulation to model approximately how stimuli on a computer monitor might look to a rat when viewing the stimuli from different distances. As can be seen from Figure 6B, when stimuli are viewed from 2 $\mathrm{cm}$ (the approximate distance from which the stimuli are viewed when making a response to the touchscreen), the image is quite clear. However if the rat were to view these stimuli from $40 \mathrm{~cm}-$ the distance at which rats are required to make their choice in some apparatus such as Grice boxes, water tanks, or jumping stands-one can see that the details of the stimuli suffer significantly. This analysis is consistent with the finding from Experiments 4 and 5 that even albino rats with very poor acuity can perform very well in this apparatus, indeed as well as non-albino rats, and that rats can discriminate photographic stimuli, which would be particularly difficult for rats to discriminate when viewed from a distance (Fig. 6B). Thus, the short viewing distance may be a major reason why rats perform so well in the touchscreen apparatus.

The proficient performance of rats using touchscreens in this study contrasts with the comparatively very poor performance reported in a recent study by Minini and Jeffery (2006). Indeed, the rats in that study were presented with a two-choice discrimination between white shapes on a black background and were unable to perform at a mean level of even $80 \%$ correct after 60 sessions of over 100 trials per session, a rate of learning that 
some might rightly consider too poor to make the touchscreen method practicable for neurobiological investigation. In contrast, in our study, rats were also presented with a two-choice discrimination between white shapes on a black background but were able to perform at a mean level of over $80 \%$ correct after fewer than six sessions of 60 trials each. Thus, the learning rates in our study and those of Minini and Jeffery (2006) differ by an order of magnitude. Two aspects of the Minini and Jeffery study are worth commenting on here. First, in our original methods paper (Bussey et al. 1994), we reported that the use of the "shelf" (see Fig. 1) forced rats to rear up and to make an effortful response to the screen, thus minimizing accidental and impulsive choices and increasing attention to the stimuli on the screen. In addition, we have found that rats perform much better using the infrared touchscreens used in this and previous studies (e.g., Bussey et al. 1994, 1997b), in which they do not have to physically press the screen, than with touchscreens that are "pressuresensitive." Minini and Jeffery did not use a shelf and used a pressure-sensitive touchscreen. The comparatively poor performance of the rats in their study suggests that these aspects of the method may be particularly important.

Once the rats in the Minini and Jeffery (2006) study had acquired the discrimination to criterion, the authors conducted a number of stimulus manipulations and concluded that rats were not using "shape information" to solve the discrimination. Specifically, they concluded that their rats tended preferentially to attend to brightness information because of the shapes protruding into the lower portions of the stimulus display. However, the fact that the rats in the study of Minini and Jeffery had such difficulty learning under the conditions used in that study raises the question of whether Minini and Jeffery's findings would generalize to other cases in which more optimal methods were used. There is already evidence that they do not. Rats using our method, even with suboptimal parameters, solve eight-pair concurrent discriminations rapidly (indeed, in many times fewer sessions than it would take to solve eight pairs sequentially) (Bussey et al. 1997b), and in such discriminations, the rats cannot simply use brightness information, as sometimes the $\mathrm{S}+$ is the brighter stimulus, and sometimes the $S+$ is the dimmer stimulus. Thus, we know that rats do not have to rely on brightness information to solve discriminations presented on a touchscreen. The finding of Experiment 5, showing that rats perform well on discriminations of photographic stimuli of the same overall shape, provides additional support for the idea that rats do not have to rely on such information to solve discriminations presented on a touchscreen.

\section{Conclusion}

In this study, we sought to manipulate parameters to optimize learning in a touchscreen apparatus. When parameters are optimized, learning can take place at a rate comparable with other, more labor-intensive methods. But there are many advantages to this method other than just minimization of labor. First and foremost is its suitability for translational research. Human patients and non-human primates are increasingly being tested using methods in which stimuli are presented on computer screens, the subject making a response to the screen via a touchscreen device (e.g., "CANTAB," Robbins et al. 1994). The rodent touchscreen method therefore provides the ability to test rodents on tasks in many cases identical to those used in humans. An additional advantage of a purely visual method is that with methods in other modalities such as olfaction, it is difficult for the experimenter to know when and where, from the rat's point of view, an odor is present or absent. In paradigms in which rats must dig in a medium such as sand laced with odors, the odor may remain present in the environment, and perhaps even on the animal, for an indefinite amount of time. Stimulus control is obviously much greater when using purely visual stimuli. A number of both nonspatial and spatial cognitive tasks are possible using purely visual stimuli in this apparatus, for both rats and mice, including single-pair discriminations (A+ vs. B - , as in this study); singlepair discrimination reversal and serial reversal (Bussey et al. 1997a; Chudasama et al. 2001; Morton et al. 2006); concurrent discrimination (three-, four-, and eight-pair) (Bussey et al. 1997a, 2001a); visuospatial conditional discrimination (if A go left, if B go right) and reversal (Bussey et al. 1997a; Chudasama et al. 2001; Janisewicz and Baxter 2003); configural discrimination tasks (Bussey et al. 1998); nonspatial nonmatching-to-sample (Bussey et al. 1994); autoshaping (discriminated Pavlovian approach; Bussey et al. 1997a; Parkinson et al. 1999, 2002; Dalley et al. 2005); and trial-unique delayed nonmatching-to-location (TUNL) (Talpos et al. 2005). All of these tasks can be administered in the same apparatus, using the same types of stimuli and responses, thus greatly facilitating comparison between tasks and allowing a "test battery" screening approach within a single apparatus, in which every test uses the same types of stimuli, responses, and rewards. The tasks are appetitive (using food reward) rather than aversive (using, e.g., electric shock or escape from cool water), which is preferable especially when testing sensitive animals such as some genetically modified rodents. In addition, the method has all of the advantages of computer automation, including ease of testing, minimized experimenter contact with the animals during testing, and consistency and accuracy in task parameters such as stimuli, responses, ITIs, and delays. Data such as reaction times are accurate and can be saved automatically into an expanding database for ease of analysis. Finally, such methods are easy to standardize, allowing for better comparison of data from different laboratories. Indeed, the touchscreen testing method has already been used to study the effects of a variety of neurobiological manipulations, including drug challenges, excitotoxic and specific neurotransmitter lesions, and drug self-administration, in both an "operant" version of the type used in this study and a "Pavlovian" version that allows the examination of Pavlovian approach learning (Bussey et al. 1997a). Thus, the flexibility and other advantages of the touchscreen method make it a particularly useful addition to the methods currently available for the cognitive testing of rodents.

\section{Materials and Methods}

\section{General methods}

\section{Subjects}

The subjects were male Lister Hooded rats (Harlan Olac) or male or female Sprague-Dawley rats (Harlan Olac), $~ 8$ - to 9-wk old and weighing $\sim 275-300 \mathrm{~g}$ at the start of the experiment, housed in groups of four in a temperature controlled room $\left(21^{\circ} \mathrm{C}\right)$ under a reversed day-night cycle (12-h light/12-h dark; lights on from 7:00 p.m.-7:00 a.m.). All testing occurred at a regular time during the dark period. The animals were fed a restricted diet of RM1(P) rat chow (Special Diet Services) that maintained them at no less than $85 \%$ of their free feeding weight throughout the experiment with water available ad libitum.

\section{Apparatus}

Preliminary training and behavioral testing were carried out in 10 automated touchscreen testing chambers (see Fig. 1A). The apparatus consisted of a standard modular testing chamber housed within a sound-attenuating box (Med Associates Inc.). The box was fitted with a 28 -volt DC fan for ventilation and masking of extraneous noise. The inner operant chamber $(30.5 \times 24.1 \times 8.25 \mathrm{~cm}$; Med Associates Inc.) consisted of a metal frame, clear Perspex walls, and a stainless steel grid floor. A pellet receptacle (magazine) attached to a $45-\mathrm{mg}$ pellet dispenser 
was situated outside of the box. A 3W houselight and tone generator (Med Associates Inc.) were fitted to the back wall of the chamber. The magazine was illuminated by a $3 \mathrm{~W}$ light bulb and fitted with photocell head entry detectors to detect the rat's presence in that area of the testing chamber.

At the end of the box opposite the magazine was a flatscreen monitor equipped with an infrared touchscreen (Craft Data Ltd., ELO Touchsystems Inc., Displaze) mediated by ELO touchscreen software (ELO Touchsystems Inc.). The use of a touchscreen that uses infrared photocells means that the rat is not required to exert any pressure on the monitor screen in order for a nose-poke to be detected; a nose-poke is registered during the rat's natural sniffing behavior toward stimuli presented on the screen. We have found that other types of touchscreen that require contact with the screen do not elicit optimal performance from rodents. A Perspex "mask" was placed over the screen; this was a sheet of Perspex that covered the screen with two response windows through which the rat could make a nose-poke toward the screen. Attached to the mask was a "shelf" extending $7 \mathrm{~cm}$ from the surface of the mask supported by springs (to prevent the rat climbing onto it). The shelf was positioned in front of the touchscreen, and the two response windows, in which stimuli were displayed, could be accessed above the mask $\sim 15 \mathrm{~cm}$ from the floor of the chamber. The effect of the shelf was to force the rat to stop, rear up, and stretch toward the stimuli with a head-on approach, thus facilitating the rat's attention to the stimuli.

\section{General behavioral procedures}

\section{Pretraining}

Rats were initially shaped to collect $45-\mathrm{mg}$ food pellets (Formula $\mathrm{P}$, Research Diets) from the food magazine. During the first session, rats were habituated to the testing chamber. Pellets were placed in the magazine and the rats left in the testing chamber for $15 \mathrm{~min}$. In the next session, the rats were trained to collect pellets that were delivered under a variable interval 30 -sec schedule together with the illumination of the magazine light and presentation of the tone. During this stage, training stimuli (40 stimuli varying in brightness, shape, and pattern) were presented on the touchscreen, one per trial in either of the two response windows for $30 \mathrm{sec}$. Multiple training stimuli were used to minimize the development of biases to particular features of stimuli. A single pellet was delivered immediately after stimulus offset. If the rat touched the stimulus, however, the stimulus disappeared and the rat was rewarded with three pellets. Completion of this stage, however, did not depend on the rat touching the stimuli on the screen, and rats were removed from the testing chamber after $30 \mathrm{~min}$ regardless of the number of trials completed.

In the next session, rats were required to respond to the stimulus presented on the touchscreen to gain reward. On each trial, a training stimulus was shown in one of the two response windows. The stimulus remained on the screen until the rat responded to it, after which the rat was rewarded with a pellet, tone, and illumination of the magazine light. This was followed by a variable ITI (initially $10 \mathrm{sec}$ ) after which the stimuli for the next trial were displayed on the screen. Once rats were successfully completing 50 trials in a 30-min session, they were required to initiate each trial. After a choice had been made, the first head entry into the magazine after the ITI resulted in the stimuli being displayed for the next trial. This meant that on every trial the rat was situated at the back of the testing chamber when the stimuli were displayed. The first head entry into the magazine during a session resulted in the stimuli being displayed for the first trial.

Once the rat was able to obtain 50 pellets within $20 \mathrm{~min}$, it was moved onto the next stage, in which punishment for incorrect responses and a correction procedure were introduced. The task now was effectively a two-choice discrimination, run in the same way as in the task proper (see below), but in this pretraining version simply required a choice between the response window containing a stimulus and the one containing no stimulus. On a given trial, a stimulus was presented on the computer screen in one of the two response windows. The rat was required to approach the touchscreen and make a response by selecting one of the two stimuli via a nose-poke. Correct responses were followed by the disappearance of the stimuli and the presentation of a pellet and tone concomitant with the illumination of the food magazine, followed by a 10-sec ITI. Incorrect responses resulted in the disappearance of the stimuli and the houselight being extinguished for a time-out period of $5 \mathrm{sec}$, followed by the ITI. A correction procedure was implemented whereby the trial was repeated until the rat made a correct choice.

\section{Two-choice discrimination task.}

Once the rats had completed pretraining, they were then trained on a two-choice visual discrimination. A pair of stimuli would appear on the screen; one stimulus was the correct $\mathrm{S}+$ and the other the incorrect $\mathrm{S}-$. A nose-poke to the $\mathrm{S}+$ resulted in a tone, magazine light, and a reward pellet. Incorrect responses resulted in a 5-sec time-out period followed by the correction procedure. Both discriminative stimuli were presented an equal number of times during a session. The left-right arrangement of the stimuli was determined pseudorandomly, with a constraint that a given stimulus could not appear on the same side of the screen on more than three consecutive trials. Performance was measured by calculating the percent correct choices per session of 100 trials.

\section{Experiment 1}

\section{Stimuli}

A range of potential stimuli were selected from Microsoft Word fonts and were processed for use in the visual discrimination task. The six stimulus pairs are shown in Figure 1B.

\section{Behavioral methods}

To determine whether the rats demonstrated a bias toward certain stimuli, a group of 24 pretrained male Lister Hooded rats were given six sessions of 100 two-choice discrimination trials as described above. A different stimulus pair was presented during each session with the same pair of stimuli presented on each of the 100 trials within a session. Stimuli were counterbalanced for order-not all rats saw all the stimulus pairs in the same order. Little learning occurs during the first session of such discriminations, thus any biases toward a particular stimulus would be reflected in the percent correct score.

\section{Experiment 2}

Twenty-four male Lister Hooded rats were trained on four different stimulus sizes. Shape stimuli were sized to fit inside squares having the following dimensions: $2.5 \mathrm{~cm}^{2}$ (size 1 ), $3.5 \mathrm{~cm}^{2}$ (size 2), $4.5 \mathrm{~cm}^{2}$ (size 3 ), and $5.5 \mathrm{~cm}^{2}$ (size 4 ). Four pairs of stimuli were used, brought forward from Experiment 1; these were pairs 1, 2, 3 , and 6 (Fig. 1B). The ITI was $5 \mathrm{sec}$. A rat would receive 10 sessions of 100 trials each of one pair, then move on to another pair, until each rat had been tested on all four pairs. The stimulus pairs were tested in the same order $(1,2,3$, then 6$)$, but the size of the stimuli in the pair was counterbalanced to control for order of presentation. Thus, every rat was tested on every pair and every size.

\section{Experiment 3}

Sixty male Lister Hooded rats were divided into eight groups, each with a different combination of the parameters ITI, trial number, and requirement to initiate trials. All rats were tested with stimulus pair 2 ("spider" and "plane") with stimulus size 4 (Fig. 1B). Rats were tested with one of two ITI s, 5 sec or 20 sec; one of two session lengths, 20 trials or 60 trials; and one of two conditions, one in which initiation was required and one in which it was not. Each of the eight possible combinations of these parameters was represented in one of the eight groups. In the cases where no initiation was required, the program would automatically present the next trial on the screen immediately after the ITI, irrespective of the rat's position in the testing chamber. Rats were tested for 22 sessions. 


\section{Experiment 4}

Ten female and nine male Sprague-Dawley rats were tested on a two-choice discrimination task using stimulus pair 2 (Fig. 1B) at size 4 . The experiment was counterbalanced such that half of the rats received one stimulus in the pair as the $S_{+}$, and the other half received the other stimulus as the $S+$. Rats received 60 trials per daily session with a requirement for initiation and an ITI of 20 sec. Rats were required to learn the discrimination to a criterion of $85 \%$ on $2 \mathrm{~d}$ consecutively. Once this was achieved, the S+ was reversed, and the previous $\mathrm{S}+$ became the new $\mathrm{S}+$. Rats were then required to learn the new $S+$ to the same criterion.

\section{Experiment 5}

Sixteen male Lister Hooded rats were tested on a two-choice discrimination task using photographic stimuli, shown in Figure 6A. The photographs were presented within two identical shapes (circles), the diameter of which was $5.3 \mathrm{~cm}$. The experiment was counterbalanced such that half of the rats received one stimulus in the pair as the S+, and the other half received the other stimulus as the S+. Rats received 60 trials per daily session with a requirement for initiation and an ITI of $20 \mathrm{sec}$.

\section{Acknowledgments}

We thank Suzanna Forwood for help with image analysis, and Lori Minini and Kate Jeffery for helpful comments on an earlier version of the paper. This work was supported by a grant from the HiQ foundation.

\section{References}

Barnet, R.A., Grahame, N.J., and Miller, R.R. 1995. Trial spacing effects in Pavlovian conditioning: A role for local context. Anim. Learn. Behav. 23: 340-348.

Brigman, J., Bussey, T.J., Saksida, L.M., and Rothblat, L. 2005. Discrimination of multidimensional visual stimuli by mice: Intraand extradimensional shifts. Behav. Neurosci. 118: 839-842.

Bussey, T.J., Muir, J.L., and Robbins, T.W. 1994. A novel automated touchscreen procedure for assessing learning in the rat using computer graphic stimuli. Neurosci. Res. Commun. 15: 103-110.

Bussey, T.J., Everitt, B.J., and Robbins, T.W. 1997a. Dissociable effects of cingulate and medial frontal cortex lesions on stimulus-reward learning using a novel Pavlovian autoshaping procedure for the rat: Implications for the neurobiology of emotion. Behav. Neurosci. 111: 908-919.

Bussey, T.J., Muir, J.L., Everitt, B.J., and Robbins, T.W. 1997b. Triple dissociation of anterior cingulate, posterior cingulate, and medial frontal cortices on visual discrimination tasks using a touchscreen testing procedure for the rat. Behav. Neurosci. 111: 920-936.

Bussey, T.J., Warburton, E.C., Aggleton, J.P., and Muir, J.L. 1998. Fornix lesions can facilitate acquisition of the transverse patterning task: A challenge for "configural" theories of hippocampal function. $J$. Neurosci. 18: 1622-1631.

Bussey, T.J., Dias, R., Amin, E., Muir, J.L., and Aggleton, J.P. 2001a. Perirhinal cortex and place-object conditional learning in the rat. Behav. Neurosci. 115: 776-785.

Bussey, T.J., Saksida, L.M., and Rothblat, L.A. 2001b. Discrimination of computer-graphic stimuli by mice: A method for the behavioral characterization of transgenic and gene-knockout models. Behav. Neurosci. 115: 957-960.

Bussey, T.J., Saksida, L.M., and Murray, E.A. 2003. Impairments in visual discrimination after perirhinal cortex lesions: Testing 'declarative' versus 'perceptual-mnemonic' views of perirhinal cortex function.
Eur. J. Neurosci. 17: 649-660.

Chudasama, Y. and Robbins, T.W. 2003. Dissociable contributions of the orbitofrontal and infralimbic cortex to pavlovian autoshaping and discrimination reversal learning: Further evidence for the functional heterogeneity of the rodent frontal cortex. J. Neurosci. 23: 8771-8780.

Chudasama, Y., Bussey, T.J., and Muir, J.L. 2001. Effects of selective thalamic and prelimbic cortex lesions on two types of visual discrimination and reversal learning. Eur. J. Neurosci. 14: 1009-1020.

Cook, R.G., Geller, A.I., Zhang, G.R., and Gowda, R. 2004. Touchscreen-enhanced visual learning in rats. Behav. Res. Methods Instrum. Comput. 36: 101-106.

Dalley, J.W., Lääne, K., Theobald, D.E., Armstrong, H.C., Corlett, P.R., Chudasama, Y., and Robbins, T.W. 2005. Time-limited modulation of appetitive Pavlovian memory by D1 and NMDA receptors in the nucleus accumbens. Proc. Natl. Acad. Sci. 102: 6189-6194.

Izquierdo, I., Medina, J.H., Bianchin, M., Walz, R., Zanatta, M.S., Da Silva, R.C., Bueno e Silva, M., Rueschel, A.C., and Paczko, K. 1993. Memory processing by the limbic system: Role of specific neurotransmitter systems. Behav. Brain Res. 58: 91-98.

Janisewicz, A.M. and Baxter, M.G. 2003. Transfer effects and conditional learning in rats with selective lesions of medial septal/diagonal band cholinergic neurons. Behav. Neurosci. 117: 1342-1352.

Kobayashi, D.T. and Chen, K.S. 2005. Behavioral phenotypes of amyloid-based genetically modified mouse models of Alzheimer's disease. Genes Brain Behav. 4: 173-196.

Markham, M.R., Butt, A.E., and Dougher, M.J. 1996. A computer touch-screen apparatus for training visual discriminations in rats. $J$. Exp. Anal. Behav. 65: 173-182.

Minini, L. and Jeffery, K.J. 2006. Do rats use shape to solve "shape discriminations"? Learn. Mem. 13: 287-297.

Morton, A.J., Skillings, E., Bussey, T.J., and Saksida, L.M. 2006. Measuring cognitive deficits in disabled mice using an automated interactive touchscreen system. Nat. Methods 3: 767.

Parkinson, J.A., Olmstead, M.C., Burns, L.H., Robbins, T.W., and Everitt, B.J. 1999. Dissociation in effects of lesions of the nucleus accumbens core and shell on appetitive Pavlovian approach behavior and the potentiation of conditioned reinforcement and locomotor activity by D-amphetamine. J. Neurosci. 19: 2401-2411.

Parkinson, J.A., Dalley, J.W., Cardinal, R.N., Bamford, A., Fehnert, B., Lachenal, G., Rudarakanchana, N., Halkerston, K.M., Robbins, T.W., and Everitt, B.J. 2002. Nucleus accumbens dopamine depletion impairs both acquisition and performance of appetitive Pavlovian approach behaviour: Implications for mesoaccumbens dopamine function. Behav. Brain Res. 137: 149-163.

Prusky, G.T., Harker, K.T., Douglas, R.M., and Whishaw, I.Q. 2002. Variation in visual acuity within pigmented, and between pigmented and albino rat strains. Behav. Brain Res. 136: 339-348.

Robbins, T.W., James, M., Owen, A.M., Sahakian, B.J., McInnes, L., and Rabbitt, P. 1994. Cambridge Neuropsychological Test Automated Battery (CANTAB): A factor analytic study of a large sample of normal elderly volunteers. Dementia 5: 266-281.

Sahgal, A. and Steckler, T. 1994. TouchWindows and operant behaviour in rats. J. Neurosci. Methods 55: 59-64.

Steckler, T. and Sahgal, A. 1995. Psychopharmacological studies in rats responding at touch-sensitive devices. Psychopharmacology 118: 226-229.

Stollnitz, F. 1965. Spatial variables, observing responses, and discrimination learning sets. Psychol. Rev. 72: 247-261.

Talpos, J.C., Dias, R., Bussey, T.J., and Saksida, L.M. 2005. The Automated Spatial Array Task, a novel method for measuring hippocampal sensitive memory in a touch-sensitive screen. Program No. 998.15. Abstract Viewer/Itinerary Planner. Society for Neuroscience, Washington, DC.

Received March 26, 2008; accepted in revised form April 24, 2008. 


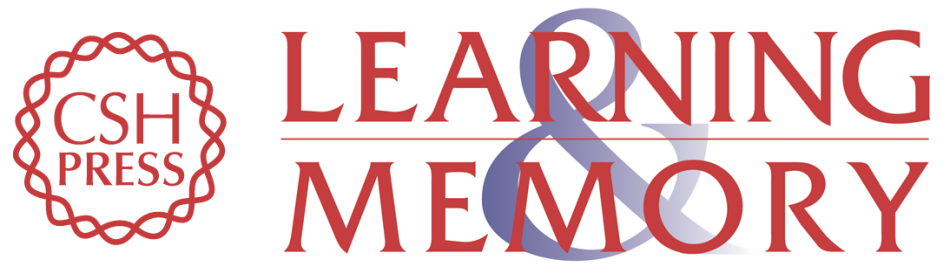

\section{The touchscreen cognitive testing method for rodents: How to get the best out of your rat}

Timothy J. Bussey, Tina L. Padain, Elizabeth A. Skillings, et al.

Learn. Mem. 2008, 15:

Access the most recent version at doi:10.1101//m.987808

References This article cites 26 articles, 5 of which can be accessed free at:

http://learnmem.cshlp.org/content/15/7/516.full.html\#ref-list-1

License

Email Alerting Receive free email alerts when new articles cite this article - sign up in the box at the Service top right corner of the article or click here. 